\title{
Pouvoir \\ Une analyse par les institutions
}

\author{
Isabelle Huault, DRM, Université Paris Dauphine \\ Bernard Leca, Groupe ESC Rouen
}

\begin{abstract}
Résumé
Les chercheurs néo-institutionnalistes se sont attachés récemment à intégrer la question du pouvoir dans leur corpus théorique. Pourtant, la plupart de ces travaux se focalisent sur le volontarisme des acteurs et sur la manière dont ils manipulent les institutions. Notre papier offre un autre point de vue en insistant sur le rôle des institutions. Nous soutenons que le processus d'institutionnalisation réfère à la manière dont des pratiques, des outils et des modèles institutionnalisés acquièrent une forme de pouvoir et modèlent les actions. Nous distinguons, pour ce faire, trois dimensions du pouvoir (pouvoir épisodique, domination et contrôle) qui correspondent à trois étapes du processus d'institutionnalisation. Lorsqu'elles sont «tenues pour acquises", les institutions ne sont plus manipulées par les acteurs de manière stratégique mais elles exercent au contraire un véritable contrôle. Nous discutons des implications de cette analyse pour la recherche en théorie des organisations et de ses articulations possibles avec la théorie critique.
\end{abstract}

\begin{abstract}
Institutional researchers have recently paid more attention to power. Yet, most of these works consider actors and the way they can manipulate institutions. This paper offers another approach by focusing on institutions. We argue that the institutionalization process is a process whereby practices, tools and patterns that are institutionalized acquire some power to shape actors' actions. We distinguish three dimensions of power, episodic power, domination and control that correspond to three steps of the institutionalization process. Once taken for granted, institutions are enforced by actors rather than manipulate by them in a strategic way. We discuss the implications for research in organizations as settings where multiple such institutionalized practices exist and possible connection with critical social theory.
\end{abstract}

\section{Introduction}

Jusqu'à une période récente, la question du pouvoir n'a reçu qu'une attention marginale dans le néo-institutionnalisme sociologique (pour une revue de littérature récente sur le sujet voir Lawrence, 2008). Ce courant théorique se concentre principalement sur l'analyse des mécanismes mimétiques par lesquels des acteurs, des individus ou des organisations, en 
viennent à se comporter tous de la même manière (DiMaggio et Powell, 1983 ; Meyer et Rowan, 1977 ; Scott, 2001 ; Tolbert et Zucker, 1983). Ces manières sont tenues pour acquises et naturelles à l'issue du processus d'institutionnalisation, de sorte qu'il ne viendrait pas à l'idée de les remettre en cause (Berger et Luckmann, 1966).

Ce n'est que récemment que la thématique du pouvoir a été considérée par les chercheurs néoinstitutionnalistes. Cette prise en compte s'est effectuée sous une forme assez conventionnelle : En analysant comment des acteurs peuvent utiliser leur pouvoir pour influencer le processus d'institutionnalisation d'une part, en spécifiant comment des acteurs sont susceptibles de recourir aux institutions pour accroître ou maintenir leur pouvoir d'autre part (par exemple, DiMaggio, 1988 ; Garud, Hardy et Maguire, 2007 ; Lounsbury, 2003). Le pouvoir est ici appréhendé dans une acception classique : soit il est exercé directement par des acteurs en l'absence d'institutions, soit les institutions sont des leviers utilisés par les acteurs pour exercer le pouvoir (voir pour le modèle sans doute le plus complet, Lawrence, Winn et Deveraux Jennings, 2001). Ainsi, les dimensions du pouvoir prises en compte s'inscrivent principalement dans l'approche 'radicale' du pouvoir proposée par Lukes $(1974,2005)$ qui constitue un classique sur ce thème.

Lukes distingue en effet trois dimensions (pour une présentation plus précise voir Annexe 1). La première concerne l'exercice ouvert du pouvoir par un acteur A sur un acteur B, ce qui conduit B à faire des choix et à agir d'une manière qu'il n'aurait pas adoptée sans l'influence de A. La deuxième renvoie au contrôle des possibilités même de décisions. Il s'agit de cas où A est capable de limiter l'étendue des problèmes qui peuvent faire l'objet de décisions de la part de $\mathrm{B}$, en contrôlant l'agenda, notamment par l'intermédiaire d'institutions. La troisième réfère à la domination exercée par $\mathrm{A}$, laquelle permet de déterminer les préférences de $\mathrm{B}$ et de l'empêcher de formuler des revendications. La domination est alors acceptée. Si les 
institutions peuvent aider à l'exercice du pouvoir de A, elles sont, dans tous les cas, des moyens d'exercice de ce pouvoir.

En utilisant le cadre néo-institutionnaliste, nous suggérons dans cet article qu'il est possible, d'ajouter des éléments à la distinction tripartite de Lukes. Ce dernier considère le pouvoir comme une relation entre des acteurs. Intégrer les institutions dans l'analyse, et le fait qu'elles soient tenues pour acquises, conduit à prendre en compte également les relations entre les institutions et les acteurs; et notamment les situations dans lesquelles les institutions elles mêmes dominent les acteurs, comme cela est le cas dans de nombreuses situations organisationnelles. Traditionnellement, l'analyse du pouvoir dans les travaux néo institutionnalistes suggère que si les institutions s'imposent aux acteurs, les acteurs peuvent également les changer. Or, cet article propose d'étendre et de compléter cette approche en intégrant les situations dans lesquelles le niveau d'institutionnalisation des pratiques est tel, que cette possibilité de changement n'existe plus, ou du moins implique un travail considérable. Cela renvoie donc à des situations dans lesquelles les institutions façonnent l'action humaine qui se contente de s'y conformer et de les reproduire.

Par exemple, la comptabilité peut être considérée comme une institution exerçant directement son pouvoir. Si l'émergence de la comptabilité à la Renaissance, peut être expliquée comme un moyen pour certains acteurs de servir leurs intérêts ${ }^{1}$ (Carruthers et Wespeland, 1991), la comptabilité constitue aujourd'hui un ensemble de pratiques et de cadrages cognitifs dont il

\footnotetext{
1 Carruthers et Wespeland (1991) rendent compte de l'émergence de la comptabilité en partie double. Ils suggèrent que celle-ci a été mis en place comme un moyen rhétorique visant à assurer convaincre certaines audiences (les investisseurs, l'église, etc.) de la légitimation des entreprises commerciales dans un contexte de changement qui correspondait à l'émergence du capitalisme entrepreneurial. Ils montrent cependant que la comptabilité a pour effet de cadrer la réalité économique. Les choses et leur sens sont fixés de manière écrite et sous forme quantitative, leur classement s'effectue selon un arrangement tabulaire ce qui conduit à cadrer cognitivement les acteurs utilisant cette méthode, et les incite à décider de manière 'plus rationnelle' en fonction de l'information telle qu'est elle est retraitée par la comptabilité.
} 
est difficile de s'affranchir. Elle constitue une pratique obligatoire, 'tenue pour acquise' et que les acteurs adoptent par obligation, plus que par calcul. Les comptables obéissent aux règles de la comptabilité, et les techniques dites de 'comptabilité créative', comme les opérations de haut de bilan, constituent des déviances hors de «l'orthodoxie comptable». Si cette institution ne sert par directement les intérêts de certains acteurs, elle n'en est pas pour autant neutre en termes de pouvoir. Elle exerce elle-même un pouvoir, c'est-à-dire qu'elle conduit des acteurs à faire des choix et à agir d'une manière qu'ils n'auraient pas adoptée sans son influence. Ce sont ces types de phénomènes, en particulier le pouvoir qu'exercent les normes comptables, que le néo institutionnalisme permet d'intégrer dans la réflexion. Il ne s'agit pas seulement de rendre compte des rapports entre des acteurs sociaux, mais entre des institutions et des acteurs sociaux. Ceci rend possible l'établissement d'un lien avec la problématique plus générale de la 'gouvernementalité', c'est-à-dire l'ensemble des procédures, les institutions, les calculs et les tactiques susceptibles d'exercer le pouvoir sur une population (Foucault, 1978), mais également le contrôle social et la nécessité de penser les instruments de ce contrôle et leurs effets (par exemple Agamben, 2006; Rancière, 1998).

L'objectif de cet article est de montrer comment l'approche centrée sur les institutions s'articule avec l'analyse de Lukes. Il est de rendre compte non seulement des rapports de pouvoir entre les acteurs sociaux, mais aussi entre les acteurs sociaux et les institutions.

La suite de l'analyse s'articule en trois étapes. Dans un premier temps, nous exposons notre cadre d'analyse et les différentes étapes du processus d'institutionnalisation (Berger et Luckmann, 1966). Nous présentons ensuite l'évolution des dimensions du pouvoir en fonction du degré d'institutionnalisation. Nous suggérons finalement que l'institutionnalisation marque la montée en puissance du pouvoir des institutions (ce que nous nommons ici contrôle social), ce qui permet de compléter le modèle de Lukes (1974) d'une part, d'infléchir l'analyse du 
pouvoir dans le néo-institutionnalisme d'autre part et, ce faisant, de situer la problématique du contrôle par les institutions, au cœur même du néo institutionnalisme organisationnel.

\section{1- Institutionnalisation et transformation du pouvoir}

Notre analyse propose une démarche liant degré d'institutionnalisation et modification des dimensions du pouvoir. Cette première partie présente la définition retenue de ces notions, ainsi que le lien qui les unit.

Nous définissons l'institutionnalisation au sens classique de Berger et Luckmann, c'est-à-dire comme un processus par lequel des pratiques deviennent tenues pour acquises (Berger et Luckamnn, 1996 [1966], voir aussi Tolbert et Zucker, 1996).

Berger et Luckmann distinguent trois mouvements :

- Le premier mouvement est l'extériorisation, c'est-à-dire l'ensemble des comportements des individus qui fait de la société un produit de l'action humaine.

- Le second mouvement est l'objectivation, résultat de la diffusion dans l'espace, et surtout dans le temps (à travers les générations) des pratiques institutionnalisées. Ces dernières en viennent à être considérées comme détentrices d'une réalité propre qu' affronte l'individu comme un fait extérieur et coercitif (Berger et Luckmann, 1996 : 84). C'est alors que la société devient une réalité objective.

- Enfin le troisième mouvement est l'intériorisation. Les pratiques institutionnalisées acquièrent pour les acteurs un statut ontologique, parce qu'ils sont socialisés au sein des institutions. Berger et Luckmann indiquent ainsi (1996: 79) «Dire qu'un segment de l'activité humaine est institutionnalisé revient déjà à déclarer que ce segment a été ordonné par le contrôle social. Les mécanismes additionnels de contrôle ne sont requis 
que dans la mesure où les processus d'institutionnalisation n'ont pas tout à fait réussi. ». L'homme devient un produit de la société.

Plus les pratiques et les manières de faire sont institutionnalisées et moins les acteurs en interrogent l'origine. Ils les considèrent comme « tenues pour acquises ». Elles sont respectées pour des raisons d'historicité ('on a toujours fait comme ça') et de contrôle social (perte de légitimité en cas de non respect) ou même légal (sanction légale pour non respect de l'institution).

Nous définissons le pouvoir dans un sens large, en reprenant la définition proposée par Lawrence (2008: 174), c'est-à-dire en tant que relation telle que les croyances et les comportements d'un acteur sont influencés par un autre acteur ou un système. Cette définition entretient une grande proximité avec celle de Lukes. Elle s'inscrit dans l'analyse relationnelle du pouvoir, défini comme une relation plutôt que comme un attribut qui pourrait être approprié et stocké. Mais elle ouvre également la possibilité que l'influence vienne non pas d'un acteur, comme chez Lukes, mais d'un système, et donc de pratiques institutionnalisées. Ceci nous conduit à dissocier pouvoir des acteurs et pouvoir des institutions.

Nous suggérons que le rapport de ces deux dimensions du pouvoir change au fur et à mesure du processus d'institutionnalisation selon une logique que nous présentons ci-après (Tableau $1)$.

Tableau 1 : Degré d'institutionnalisation et dimension du pouvoir

\begin{tabular}{|c|c|c|c|}
\hline $\begin{array}{l}\text { Degré } \\
\text { d'institutionnalisation }\end{array}$ & $\begin{array}{c}\text { Extériorisation } \\
\text { (Pas d'institution) }\end{array}$ & $\begin{array}{c}\text { Objectivation } \\
\text { (Institutions présentes) }\end{array}$ & $\begin{array}{c}\text { Intériorisation } \\
\text { Institutions tenues pour } \\
\text { acquises (statut } \\
\text { ontologique) }\end{array}$ \\
\hline
\end{tabular}




\begin{tabular}{llll}
$\begin{array}{l}\text { Pouvoir } \\
\text { discrétionnaire }\end{array}$ & Important & $\begin{array}{l}\text { Limité (le dominant } \\
\text { comme le dominé sont } \\
\text { limités par l'institution) } \\
\text { Contrôle }\end{array}$ & $\begin{array}{l}\text { Absent (le contrôleur se } \\
\text { contente d'exercer le } \\
\text { contrôle) } \\
\text { Contrôle }\end{array}$ \\
\hline Type de pouvoir & $\begin{array}{l}\text { Pouvoir par les acteurs : } \\
\text { Pouvoir épisodique }\end{array}$ & $\begin{array}{l}\text { Pouvoir par les acteurs } \\
\text { s'appuyant sur les } \\
\text { institutions : Domination }\end{array}$ & $\begin{array}{l}\text { Pouvoir par les } \\
\text { institutions : Contrôle }\end{array}$ \\
\hline $\begin{array}{l}\text { Nature de l'agence } \\
\text { Qui exerce le } \\
\text { pouvoir? } \\
\text { Sources de pouvoir }\end{array}$ & $\begin{array}{l}\text { Agent politique } \\
\text { Tous les acteurs }\end{array}$ & $\begin{array}{l}\text { Agent politique } \\
\text { Rominants }\end{array}$ & $\begin{array}{l}\text { Corps, objet de police } \\
\text { Institutions }\end{array}$ \\
\hline
\end{tabular}

La phase d'extériorisation correspond à une situation d'absence d'institutions, c'est-à-dire de règles et de routines. Les acteurs exercent un pouvoir mais qui ne repose que sur leurs seules relations épisodiques et discrétionnaires.

La phase d'objectivation correspond à l'émergence d'institutions. Celles-ci contraignent le comportement des acteurs qui sont tenus de s'y conformer, sous peine de subir les sanctions sociales ou légales associées à leur non respect. Ces institutions peuvent être utilisées par les acteurs pour assurer et stabiliser leur pouvoir. Elles servent des pratiques de domination par lesquelles des acteurs dominants usent de ces institutions pour maintenir et exercer leur pouvoir sur les dominés.

La phase d'intériorisation correspond à l'appropriation totale par les acteurs des institutions, c'est-à-dire à celle, lors de laquelle les acteurs n'ont plus de réflexivité vis-à-vis des institutions. Dans cette phase, la manipulation stratégique des institutions n'est plus possible ou ne l'est que très marginalement. Non seulement la déviance entraînerait des sanctions pour violation de l'ordre établi, mais surtout l'acteur ne songe guère à remettre en cause celui-ci. Ce sont les institutions qui imposent aux acteurs les modifications des croyances et des comportements.

Avec l'institutionnalisation, ce sont des règles qui se substituent aux rapports inorganisés. Au fur et à mesure que ces règles impersonnelles deviennent 'tenues pour acquises', elles réduisent le pouvoir discrétionnaire des acteurs jusqu'à le rendre caduque. Nous suggérons 
que la montée en puissance du pouvoir des institutions marque fondamentalement le processus d'institutionnalisation.

Nous détaillons ce processus dans les trois parties suivantes de l'article.

\section{Au commencement était le pouvoir épisodique...}

Revenir sur le processus d'institutionnalisation implique, à l'instar de Berger et Luckmann, de débuter par le constat de l'absence d'institutions. Le pouvoir repose alors sur des relations interpersonnelles qu'aucune structure institutionnalisée ne contraint. Il est épisodique.

Ce type de situation n'existe pas en tant que tel, dans la mesure où existe toujours un encastrement institutionnel et culturel antérieur à l'action des individus (Leca et Naccache, 2006). Il demeure cependant des situations dans lesquelles cet encastrement est moins important que dans d'autres, c'est-à-dire des situations dans lesquelles les institutions sont affaiblies et exercent une influence moindre. C'est notamment le cas des pays en transition institutionnelle. En analysant les situations de la Chine lors de son passage au capitalisme, ou des ex pays du bloc de l'Est, Peng (2000) montre que dans des contextes où les « institutions » officiellement en place ne fonctionnent plus, les relations interpersonnelles prennent le relais. C'est au sein même de ces relations que se situe le pouvoir.

De nombreux travaux et courants théoriques en gestion ont exploré le pouvoir lié aux relations interpersonnelles. Ils indiquent que le pouvoir dépend de l'importance des ressources, de la maîtrise de l'incertitude, et de la position occupée dans les réseaux sociaux.

Les ressources détenues constituent une première forme de pouvoir. La théorisation par Pfeffer et Salancik (1978, notamment p. 39-61) de la dépendance aux ressources souligne que les relations de pouvoir sont marquées par la capacité des acteurs à contrôler l'accès à des 
ressources dont les autres acteurs ont besoin. Dès lors qu'il y a asymétrie de ressources, il y a relation de pouvoir. On retrouve une telle situation dans le cas des banques ou des investisseurs qui peuvent prendre un poids déterminant dans la définition de la stratégie des entreprises endettées et exercer ainsi une influence directe sur celles-ci. La perspective behavioriste de Cyert et March (1963) insiste également sur l'importance des ressources. Ces auteurs postulent que les individus agissent pour défendre et accroître leur part dans les processus d'allocation de ressources au sein des organisations. Leur analyse appréhende le fonctionnement d'une organisation comme le fruit de processus politiques et d'un ajustement entre intérêts divergents. L'organisation est ainsi une véritable «arène » constituée de coalitions politiques. C'est en se coalisant que les individus peuvent espérer accroître leur contrôle des ressources.

Les théoriciens de l'analyse stratégique (Crozier et Friedberg, 1977) proposent une analyse complémentaire des relations de pouvoir, en soulignant l'importance de la capacité à créer et à contrôler de l'incertitude. Face aux règles de l'organisation, les acteurs s'aménagent des marges de manœuvre qui correspondent à autant de zones d'incertitude leur conférant potentiellement du pouvoir. Dans cette acception, les acteurs sont libres, chacun pouvant jouer avec les règles, de façon relativement imprévisible. Le pouvoir d'un individu ou d'un groupe est dès lors fonction de l'ampleur de la zone d'incertitude que l'imprévisibilité de son propre comportement lui permet de contrôler face à ses partenaires. Crozier et Friedberg mettent en évidence plusieurs sources d'imprévisibilité : l'expertise professionnelle, le contrôle du système hiérarchique ou le positionnement de marginal-sécant. Chaque individu dispose, selon ses ressources, de marges de manœuvre qui autorisent des adaptations personnelles dans un système qui n'est jamais donné d'avance. Ce dernier résulte de coopérations interindividuelles, dans lesquelles chacun essaie de faire triompher ses propres intérêts, de manière opportuniste. 
Enfin, l'analyse des réseaux sociaux fournit une autre compréhension des relations de pouvoir. Burt (1992) a mis en évidence l'importance de la centralité des acteurs comme facteur explicatif du pouvoir. Il est possible de distinguer différents types de centralité (Freeman, 1979), qui toutes ont une influence sur le pouvoir des acteurs. La centralité locale correspond à la proximité d'autres membres du réseau, et la centralité globale, à la centralité dans l'ensemble du réseau. La centralité d'intermédiation renvoie à la capacité de contrôler les flux d'information dans le réseau. Ceci implique d'être destinataire de ces informations mais également de ne pas être «contournable» (Burt, 1992). Les acteurs ayant une forte centralité et n'étant pas contournables car tous les liens passent par eux, sont dans une posture privilégiée car leur retrait du réseau entrainerait la disparition de celui-ci. Cette situation leur confère une position de pouvoir privilégiée. La centralité de proximité, c'est-à-dire la vitesse à laquelle on peut contacter les autres membres du réseau permet également d'accroître son pouvoir.

Ces différentes analyses du pouvoir ne prennent pas en compte les institutions. Elles sont compatibles avec la première dimension du pouvoir défini par Lukes (voir Annexe 1). La mise en place d'institutions permet l'émergence d'une autre dimension, plus complexe du pouvoir : la domination.

\section{3. ... vint la domination...}

La mise en place progressive des institutions a pour effet de réduire les éléments servant de base au pouvoir inter personnel que sont l'incertitude, la maîtrise de l'accès aux ressources et les réseaux. En effet, les institutions réduisent l'incertitude en affectant à chacun un rôle dans les interactions sociales. C'est même l'une de leurs fonctions premières (DiMaggio et Powell, 1991). La reproduction réciproque de certains comportements admis réduit l'incertitude mais 
également les marges de manœuvre des individus. Les institutions structurent les pratiques d'accès aux ressources. Celles-ci ne sont plus strictement discrétionnaires, car la mise en place d'institutions consiste précisément à fixer des critères selon lesquels les ressources sont allouées. Ainsi, les subventions publiques ou la certification ne sont pas accordées de manière totalement discrétionnaire, mais en fonction de critères institutionnalisés. Enfin, la structuration des relations sociales provoquée par la mise en place des institutions a pour effet de réduire l'importance des réseaux sociaux. En d'autres termes, lorsque des règles s'appliquent, la question de savoir qui l'on connaît devient moins importante.

L'émergence d'institutions en posant des règles contraignantes entraîne la limitation du pouvoir discrétionnaire. Elle engendre également une mutation de la forme du pouvoir. Car si le pouvoir épisodique fondé sur les relations directes entre les acteurs est réduit, ceci ne signifie pas pour autant que le pouvoir disparaisse.

Marx le premier a montré que l'existence d'un système institutionnel stable, souvent présenté en philosophie politique comme limitant le pouvoir des puissants a également (voire principalement) pour effet d'assurer le maintien de relations de pouvoir asymétriques et le pouvoir de certains acteurs. Les institutions servent les puissants. Ceux-ci façonnent les institutions et appuient leur pouvoir sur celles-ci et non plus seulement sur des relations sociales épisodiques. Il s'instaure alors des relations asymétriques durables entre les dominants et les dominés.

L'idée de domination suggère que si les règles s'appliquent à tous, elles servent les dominants. La lutte pour la domination dans une entreprise ou dans un champ organisationnel est une lutte pour la définition, ou la redéfinition, des institutions. Des travaux néo- 
institutionnalistes récents étudient ainsi comment une coalition dominante dans un champ 'travaille' les règles du champ pour les adapter en fonction des rapports de force en son sein. Ainsi, Greenwood, Suddaby et Hinings (2002) révèlent comment les grandes firmes d'audit agissent au sein des associations professionnelles pour changer les règles existantes dans un sens qui leur soit favorable. Levy et ses collègues (Levy et Egan, 2003 ; Levy et Scully, 2007) mobilisent la notion d'hégémonie en s'inspirant des travaux de Gramsci pour rendre compte du travail politique mené par les organisations dominantes pour maintenir ou modifier les règles dans un champ. Deux dimensions stratégiques se combinent. D'une part, le travail sur la définition des règles qui déterminent qui sont les dominants et qui sont les dominés. D'autre part, le travail sur le pouvoir discrétionnaire laissé aux dominants par ces règles. Fligstein (1997) suggère que les dominants peuvent utiliser l'autorité directe et le cadrage (framing) des actions pour convaincre les dominés qu'ils ont intérêt à maintenir les choses en l'état. Il rattache les actions de ces mêmes dominés aux deuxième et troisième dimensions du pouvoir chez Lukes.

A ce stade, il existe des institutions dont le non respect est sanctionné mais qui ne sont pas entièrement intériorisées. Les dominants les promeuvent et entreprennent de les maintenir non pas parce qu'ils les tiennent pour acquises, mais parce qu'elles servent clairement leurs intérêts. L'enjeu pour ces acteurs dominants est de faire en sorte que les dominés considèrent ces règles comme légitimes (Weber, 1995) et consentent de ce fait au maintien de la domination.

La domination se reproduit alors avec le consentement des dominés. Ceux-ci acceptent de reproduire des comportements et de respecter des règles qui ne les favorisent pas. S'interrogeant sur les raisons pour lesquelles les ouvriers travaillent si dur pour accroître la productivité dans un atelier, alors que cet accroissement bénéficie bien plus aux propriétaires 
de l'entreprise qu'à eux mêmes, Burawoy (1979) montre la prégnance des institutions dans la 'fabrication du consentement'. Il insiste notamment sur l'importance du paiement à la pièce, de la mobilité interne et de la négociation collective comme autant de processus institutionnalisés qui donnent le sentiment aux ouvriers qu'ils sont associés à la prospérité de l'entreprise et à son avenir, alors que ces processus sont conçus pour que cette association soit mineure. Ce qui est en jeu, c'est la «fabrique du consentement» des ouvriers à leur propre domination (Burawoy, 1979).

\section{4. ... puis le contrôle.}

La dernière étape est le point où les institutions deviennent intériorisées et tenues pour acquises (taken-for-granted) par tous les acteurs. Ces institutions acquièrent alors un statut ontologique, : Elles sont considérées comme faisant partie de ce qui est «naturel» par tous les acteurs. Il n'est alors plus possible de retrouver les indices de leur construction sociale. Butler indique (2005: 59) «...le caractère institutionnel - je dirais même l'ancrage institutionnel - de l'ontologique au point que l'on ne peut plus percevoir la trace de l'institution dans l'effet ontologique qu'elle produit ». Les acteurs reproduisent des pratiques institutionnalisées, sans être capables d'expliquer leur signification. Swidler (2001) suggère même que cette confusion favorise la durabilité des pratiques. Les institutions participent alors directement au cadrage cognitif des acteurs. Elles acquièrent une capacité à influencer les processus cognitifs des dominés comme des dominants. Et plus les institutions sont tenues pour acquises, moins les acteurs peuvent exercer un pouvoir discrétionnaire. En ce, le processus d'institutionnalisation est un processus de dé-subjectivation, c'est-à-dire que les acteurs sont de moins en moins considérés comme sujets et de plus en plus considérés comme objets (Agamben, 1997). Les acteurs dominants continuent à exercer leur autorité mais sans 
réflexivité vis-à-vis d'institutions qu'ils considèrent comme naturelles. Ils se contentent d'appliquer les règles.

Le gouvernement qui s'impose alors, est celui des institutions qui influencent les acteurs, leur comportement et leur cognition. Contrairement à l'étape précédente, ces institutions sont enactées pour elles même et non parce qu'elles servent la domination de certains.

Les pratiques et les règles intériorisées s'émancipent de leurs promoteurs. C'est ce que DiMaggio souligne lorsqu'il indique «L'institutionalisation en tant que résultat place les structures et les pratiques hors d'atteinte des intérêts et des jeux politiques $»^{2}(1988: 13$, italiques par l'auteur). Une fois intériorisées, elles ne peuvent pas facilement être modifiées, y compris par ceux là même qui les ont mises en place. Ces institutions figent les interactions et les modifier, entraînerait une destruction des routines, un accroissement de l'incertitude et la déstabilisation des interactions. Une fois les règles tenues pour acquises, elles perdurent. Enfin, les institutions cadrent les raisonnements des acteurs (Hasselbladh et Kallinikos, 2000). Zuckerman (1999) montre ainsi l'influence des catégories sur le jugement des analystes financiers. Ceux-ci utilisent ces catégories pour évaluer les entreprises. Face à des firmes qui n'entrent pas « dans les cases », ces analystes sont méfiants et tendent à leur faire payer cette non-conformité en les sous évaluant. Déjean, Gond et Leca (2004) rendent compte de la manière dont une mesure de performance sociétale des entreprises qui se diffuse et s'institutionnalise parmi les gérants de fonds socialement responsables influence les pratiques et la manière dont les gérants définissent la responsabilité sociale, font sens de leur métier et établissent leur identité.

Les institutions constituent finalement des systèmes de définition et de hiérarchisation (Rancière, 1998). Les acteurs cessent d'être considérés comme doués de capacités

\footnotetext{
${ }^{2}$ Institutionalization as an outcome places organizational structures and practices beyond the reach of interest and politics.
} 
« d'agence », c'est-à-dire d'actions autonomes non guidées par les institutions, pour se voir affectés à des rôles (Berger et Luckmann, 1966) dont le respect doit être contrôlé et sanctionné.

Les acteurs sont alors considérés comme des objets de contrôle. Les institutions établissent des rôles. Est légitime ce qui correspond au rôle, est illégitime ce qui s'en éloigne. Elles hiérarchisent selon le niveau de conformité. Les institutions assignent également des places (Rancière, 1995), et façonnent les identités. Ces mécanismes fixent des définitions mais assurent également des suivis et des vérifications des comportements pour s'assurer de leur conformité à la règle.

Il y a bien pouvoir, puisque les acteurs agissent en fonction de l'influence d'un élément extérieur, les institutions, sans lesquelles ils se comporteraient différemment. Ce pouvoir procède des institutions. Il ne s'agit pas d'un pouvoir discrétionnaire et il s'applique à tous les acteurs répartis selon leur rôle.

Ce pouvoir est d'ailleurs d'autant plus puissant qu'il est rassurant. En rendant les comportements prévisibles, les institutions réduisent la liberté des acteurs mais diminuent également l'incertitude et favorisent la coordination. Elles sont donc, pour cette raison, utiles à des acteurs qui sont 'risk adverse' et appréciées de ceux-ci.

Les autres sources de pouvoir perdent de leur importance. Ainsi, Lianos (2003 : 443) suggèret-il encore que l'accumulation de capitaux permettant d'obtenir une position dominante (au sens de la première dimension du pouvoir de Lukes), est moins valorisée, car tous sont 'égaux devant l'institution'. Comme il l'indique, « il devient de plus en plus difficile dans un tel contexte de mobiliser des ressources propres à la stratification sociale, afin de différencier par rapport aux soupçons et effets du contrôle ; la diminution de cette marge de négociation occasionne un déclin de la motivation pour acquérir de telles ressources ; et par là-même, un désengagement par rapport à l'appartenance sociale ». Il existe encore des dominants, mais 
ceux-ci n'exercent plus de pouvoir discrétionnaire, ils remplissent le rôle que les institutions leur affectent. L'important se trouve dans l'arrangement institutionnel des différentes techniques mises en place, et non dans le travail des acteurs. Lianos (2003: 443, italiques pour l'auteur) ajoute que « en examinant le support axiologique des normes impliquées, on trouve que ces dernières ne correspondent pas à des valeurs mais s'inscrivent dans un plexus administratif, gestionnaire et technique bâti comme un lieu de consensus entre l'institution et les usagers à propos de l'efficacité de leur interaction. On s'éloigne donc de façon inédite de la génération de conformité par des réseaux relationnels, désormais substitués par la jonctionnalité seule de l'institution et des systèmes qui la servent ». Il n’y a donc pas de valeur ou d'idéal moral sous jacent, ou celui-ci a disparu dans le processus d'intériorisation. Les institutions perdurent parce que c'est la manière traditionnelle de faire, et que tout le monde la suit.

De nombreux auteurs contemporains soulignent que ce type de pouvoir est particulièrement présent dans les sociétés contemporaines (par exemple Agamben, 2006; Rancière, 1998). Lianos (2003: 434) indique qu'« il ne semble pas difficile de soutenir que la plus grande partie de l'acheminement du comportement ne provient pas dans les sociétés capitalistes d'aujourd'hui de réseaux relationnels de socialité mais des cadres, actes et activités institutionnels ». Ce développement s'effectue également dans les entreprises. La multiplication des certifications, des dispositifs de qualité totale, de reporting, la diffusion de pratiques qui sont essentiellement adoptées pour se conformer aux exigences de l'environnement, « faire comme les autres » et accroître sa légitimité (par exemple Covaleski, Dirsmith et Samuel, 1996 ; Westphal, Gulati et Shortell, 1997), font de l'entreprise un lieu propice à l'analyse du pouvoir des institutions tenues pour acquises. 
Les systèmes comptables constituent, peut-être, les meilleurs exemples de pratiques institutionnalisées qui exercent un pouvoir sur les acteurs au sein des entreprises. Scott (2003 : 139) indique que « les systèmes comptables sont parmi les conventions les plus importantes connectant les systèmes de croyance institutionnellement définis avec les activités techniques». La comptabilité constitue une manière d'assurer la coordination entre différents groupes d'acteurs qui utilisent la même norme (banquiers, dirigeants, contrôleurs de gestion, etc.). Son maintien ne sert pas directement un groupe dominant, mais celui, plutôt subalterne, des comptables. Les comptables assurent le maintien rituel des normes et s'assurent que les autres membres de l'organisation s'y conforment. L'analyse néo-institutionnelle permet de prendre en compte ce type de pouvoir qui nous semble être exclu de l'approche traditionnelle synthétisée par Lukes.

La question n'est plus alors celle du pouvoir comme exercice discrétionnaire mais celle du contrôle social et de la «gouvernementalité » qui permettent d'exercer le pouvoir sur une population. A la suite des travaux de Foucault (1978), Rancière (1998) et Agamben (2006) insistent sur l'importance de ces dispositifs. Le cœur de l'interrogation n'est plus guère celle des rapports entre les acteurs mais plutôt des dispositifs institutionnalisés en place, et la façon dont ils influencent le comportement.

\section{Discussion et conclusion}

Le but de cet article a été de proposer une grille d'analyse pour mieux rendre compte de la manière dont les dimensions du pouvoir s'articulent avec le degré d'institutionnalisation. Nous avons suggéré que l'institutionnalisation entraîne une diminution du pouvoir fondé sur les relations interpersonnelles et un accroissement du pouvoir des institutions qui deviennent 
tenues pour acquises à l'issue du processus. A la suite de Berger et Luckmann, nous distinguons trois étapes dans le processus d'institutionnalisation qui correspondent à trois dimensions du pouvoir: Le pouvoir épisodique fondé sur les relations interpersonnelles en l'absence d'institutions, la domination fondée sur les institutions qui assurent aux dominants un pouvoir stable, et le contrôle qui correspond au respect par les acteurs des rôles que leur fixent les institutions et donc au pouvoir de celles-ci.

Notre contribution s'articule dès lors autour de quatre axes principaux.

En premier lieu, notre analyse s'inscrit dans la lignée de celle de Lukes mais l'étend. Alors que cet auteur rend compte du pouvoir épisodique ainsi que des différentes formes de domination, il ne s'intéresse pas au pouvoir des pratiques institutionnalisées en tant que telles. Il les considère surtout comme susceptibles de servir à la domination d'autres acteurs. Or, prendre en compte cette dimension peut enrichir les recherches actuelles sur le pouvoir. Se pencher sur des dimensions du pouvoir qui ne sont pas manipulées stratégiquement par les acteurs mais qui proviennent plutôt du simple respect des normes en place, permet d'explorer des formes ordinaires de pouvoir d'autant plus fréquentes, que les pratiques et procédures institutionnalisées le sont.

En deuxième lieu, la grille proposée entre en résonance avec le programme de recherche originel du néo institutionnalisme sociologique. Elle permet de renouer avec lui et d'infléchir l'analyse actuelle du pouvoir par les auteurs se situant dans ce courant théorique. Le néo institutionnalisme s'intéresse initialement à la diffusion par mimétisme des institutions et suggère qu'une fois ces institutions en place, elles contraignent les actions (Zucker, 1977). Il nous apparaît que l'étendue du pouvoir des institutions « tenues pour acquises » a fait l'objet de peu d'études. Le courant néo institutionnaliste a réhabilité l'acteur stratégique à la fin des 
années quatre-vingt grâce à la notion d'entrepreneur institutionnel, quitte même à faire de celui-ci un héros ou un démiurge capable de remettre en cause toutes les institutions (pour une revue critique Garud et al., 2007). De même, la réintroduction actuelle de la problématique du pouvoir dans le néo institutionnalisme tend à mettre en avant le rôle des acteurs et à présenter les institutions comme des instruments de domination dans les mains de ceux-ci. Si ces évolutions enrichissent incontestablement le cadre théorique, ce dernier pourrait être utilement complété par une vision moins instrumentale des institutions. Cette conception, plus conforme au programme de recherche initial, prendrait en compte la manière dont les institutions influencent le comportement des acteurs, dominants comme dominés, que ceux-ci en soient conscients ou non. Cette analyse conduit dès lors à insister sur l'importance de l'étude du contrôle pour le néo-institutionnalisme.

En troisième lieu, l'importance donnée au contrôle peut permettre de lier des travaux critiques en théorie des organisations avec les travaux menés aujourd'hui en théorie sociale sur les dispositifs de contrôle. Des auteurs comme Agamben (2006), Lianos (2003) ou Rancière (1997) insistent sur l'importance de ces dispositifs en tant que tels. Ainsi, Rancière (1997) oppose-t-il le politique, qui s'appuie sur des dispositifs de gouvernement pour rassembler les hommes, obtenir leur consentement, les hiérarchiser, et repose sur la police de leurs mœurs, et la politique qui consiste à remettre en cause ce fonctionnement. Tous ces auteurs s'interrogent sur le consentement des individus à abdiquer une partie de leur liberté pour des institutions et des règles restreignant leur autonomie mais qui leur garantissent la sécurité. L'institution n'est pas seulement aliénante en effet, en ce qu'elle réduit voire supprime le pouvoir discrétionnaire et la liberté qui l'accompagne. Elle est également, pour cette même raison, rassurante. Si chacun se conforme à la manière de faire, jugée légitime, le comportement de tous devient prévisible. En réduisant la liberté, l'institution accroît la sécurité. En rendant le comportement 
de chacun prévisible, elle permet de réduire l'incertitude, de routiniser les comportements et de favoriser la 'cognition automatique', c'est-à-dire toutes ces situations dans lesquelles il n'y a pas d'inconnu et où il suffit de suivre la procédure et de faire 'comme on doit faire' sans avoir à réfléchir. En cela, les règles elles-mêmes, en tant que leur usage s'impose aux gouvernants comme manière 'légitime' de faire, doivent être précisément étudiées. Cette situation invite à s'intéresser au politique autant qu'à la politique, et aux 'dispositifs' de ce politique (Agamben, 2006).

Si l'on admet que les organisations sont des lieux dans lesquels existent de très nombreuses procédures qui régissent les comportements des individus, que celles-ci sont nécessaires pour assurer la coordination des acteurs, réduire l'incertitude, accroître la sécurité et favoriser la rapidité d'exécution; si l'on admet également qu'un grand nombre d'entre elles ne font pas l'objet de remises en cause car elles sont souvent 'tenus pour acquises', nous suggérons que cette voie peut être particulièrement féconde pour étudier les organisations. Il ne s'agit plus alors de rechercher qui se cache derrière les pratiques institutionnalisées et comment cette personne, ou ce groupe, les manipule à son bénéfice, mais de rendre compte du pouvoir de la norme et de la manière dont elle se déploie. Agamben (2006) souligne qu'analyser ces dispositifs, et la manière dont ils affectent les comportements et les vies des acteurs consiste à étudier leur gestion et leur management. Ceci suggère que la recherche en gestion peut constituer le lieu de l'analyse de ces pratiques institutionnalisées et tenues pour acquises, et la manière dont elles façonnent les comportements humains. Les mesures, les processus de contrôle, les normes comptables, les techniques managériales sont autant d'objets qu'il convient d'aborder non pas seulement en ce qu'ils serviraient une stratégie délibérée, mais en ce qu'ils constituent des institutions qui imposent des cadres cognitifs aux membres des organisations, quel que soit leur degré hiérarchique. 
Enfin, notre analyse peut également contribuer au développement des travaux critiques en gestion. Il suggère que le niveau d'institutionnalisation des pratiques, des routines ou de toute autre forme d'institution doit être pris en compte pour comprendre les possibilités et les processus d'émancipation et de changement. Plus une institution est tenue pour acquise, plus l'émancipation des acteurs est difficile, car ceux-ci la considèrent comme faisant partie de l'ordre naturel. Le travail d'émancipation devient alors un travail de réflexivité et de dénaturalisation des institutions. Des institutions considérées comme naturelles sont en fait des constructions sociales susceptibles d'être ébranlées par les acteurs eux-mêmes. Encore faut-il que les acteurs s'en rendent compte. La capacité des acteurs à dépasser le confort des routines cognitives (DiMaggio, 2002), et l'acquisition d'une réflexivité indispensable, constituent autant de conditions pour s'émanciper des pratiques institutionnalisées. Ce processus est celui qui autorise l'émergence d'une prise de conscience du caractère socialement construit et donc transformable des institutions, et qui permet d'envisager une critique productive de celles-ci. Le développement d'une telle réflexion critique est un préalable au développement d'une praxis au sens marxiste (Marx, 1987), c'est à dire d'une articulation entre l'analyse critique des cadres existants, la recherche d'alternatives, et une action impliquant la mobilisation et l'action collective (Benson, 1977). Elle est notamment favorisée par les contradictions qui peuvent surgir entre les institutions, ou lorsque celles-ci apparaissent comment particulièrement inadaptées pour répondre aux changements du contexte (Seo et Creed, 2002).

Ce processus dévoile les mécanismes de domination qui sont naturalisés par le biais des institutions. L'émancipation, et le changement peuvent alors être envisagés comme des processus de retour vers le pouvoir discrétionnaire, et en ce sens de « désinstitutionnalisation ». 


\section{Références bibliographiques}

Agamben, G. Homo Sacer, le pouvoir souverain et la vie nue, Seuil, 1997.

Agamben, G. Qu'est ce qu'un dispositif? Rivages, 2006.

Benson, J.K. "Organizations: a dialectal view”Administrative Science Quarterly, Vol 22, 1977, p.1-21.

Berger P., Luckmann T. La construction sociale de la réalité. Armand Colin, 1996 (Ed. Originale 1966).

Burawoy, M, Manufacturing Consent. Chicago: University of Chicago Press, 1979.

Burt R., Structural holes: The social structure of competition, Cambridge, Harvard University Press, 1992.

Butler, J., Gender Trouble: Feminism and The Subversion of Identity. New York : Routledge, 1990.

Butler J., Humain, inhumain, Le travail critique des normes, Editions Amsterdam, 2005.

Carruthers, B. G., et W.N. Espeland. "Accounting for Rationality: Double-Entry Bookkeping and the Rhetoric of economic Rationality". American Journal of Sociology, Vol 97, n¹, 1991, p.31-69.

Christensen, S., Karnoe, P., Pedersen, J.S. \& Dobbin, F. (Eds.). "Action in institutions", American Behavioral Scientist, Vol 40, n4, 1997, p.389-538.

Clegg S., Frameworks of power, London : Sage, 1989.

Cooper D, Ezzamel M., and Willmott H., "Examining 'Institutionalization", in R. Greenwood, C. Oliver, R. Suddaby et K. Shalin-Andersson, The Handbook of Organizational Institutionalism, Sage, 2008.

Covaleski, M. A., Dirsmith, M. W. \& Samuel, S. "Managerial accounting research: The contributions of organizational and sociological theories", Journal of Management Accounting Research, 1996, Vol 8, n 1, p.1-35.

Crozier M., Friedberg E, L'acteur et le système, Paris : Seuil, 1977.

Cyert R., March J., A behavioral theory of the firm, Englewood Cliffs, NJ : Prentice Hall, 1963.

Dahl R., “The concept of power”, Behavioral Science, Vol 20, 1957, p.201-215.

Déjean F. Gond, J.P and Leca, B., 'Measuring the Unmeasured: An Institutional Entrepreneur Strategy in an Emerging Industry' Human Relations, Vol 57, nº6, 2004, p. 741-764. 
DiMaggio, P., "Interest and agency in institutional theory", in L. Zucker (ed), Institutional patterns and organizations. Cambridge, MA: Ballinger, 1988, p.3-22.

DiMaggio, P. and Powell, W. "The Iron Cage Revisited: Institutional Isomorphism and Collective Rationality in Organizational Fields". American Sociological Review, Vol 48, 1983, p.1750-1762.

DiMaggio, P., and Powell, W. "Introduction", in W. W. Powell and P. J. DiMaggio (Eds), The New Institutionalism in Organizational Analysis. Chicago: Chicago University Press, 1991, p.1-38.

DiMaggio, P. "Why Cognitive Sociology Needs Cognitive Psychology." in Karen Cerulo (ed.), Culture in Mind: Toward a Sociology of Culture and Cognition, London: Routledge, 2002.

Fligstein, N. « Social Skill and Institutional Theory » American Behavioral Scientist, Vol 40, $\mathrm{n}^{\circ} 4,1997$, p.397-405.

Foucault, M. Les mots et les choses, Paris, Gallimard, 1966.

Foucault M., Surveiller et punir, Paris, Gallimard, 1975.

Foucault, M. Sécurité, Territoire, Population. (Cours au Collège de France 1977-178), Hautes Etudes. Gallimard/Seuil, 1978.

Garud, R. Hardy C., and Maguire S., "Institutional Entrepreneursip as Embedded Agency: An Introduction to the Special Issue", Organization Studies, Vol 28 , n7, 2007, p.957-969.

Greenwood, R, Suddaby, R. \& Hinings, C.R. « Theorizing change: The role of professional associations in the transformation of institutionalized fields ». Academy of Management Journal, Vol 45, n²1, 2002, p.58-80.

Hasselbladh, H. et Kallinikos, J., "The Project of Rationalization: A Critique and Reappraisal of Neo-Institutionalism in Organization Studies" Organization Studies, Vol 2, n 4, 2000, p. 697-720.

Hobsbawm, E. et Ranger, T., (Eds.). The Invention of Tradition. Cambridge: Cambridge University Press, 1984.

Lawrence T., "Power, Institutions and Organizations", in Greenwood R, Oliver C., Sahlin K., Suddaby R, The Sage Handbook of Organizational Institutionalism, 2008, p.170-198

Leca, B. et Naccache, P. "A Critical Realist Approach to Institutional Entrepreneurship". Organization, Vol 13, n5, 2006, p.627-651.

Levy, D. et Egan, D. "A Neo-Gramscian Approach to Corporate Political Strategy: Conflict and Accomodation in the Climate Change Negociation" Journal of Management Studies, Vol $40, \mathrm{n}^{\circ} 3,2003, \mathrm{p} .803-829$. 
Levy, D. et Scully, M. “The Institutional Entrepreneur as Modern Prince: The Strategic Face of Power in Contested Fields' Organization Studies, Special Issue, Vol 28, n²7, 2007, p.971991.

Lianos, M. «Le contrôle social après Foucault », Surveillance \& Society, Vol 1, n³, 2003, p.431-448.

Lounsbury, M. "Power and Institutions. The Problem of Order Revisited : Towards a more Critical Institutional Perspective", in Westwood R. and Clegg S., Debating Organizations, Chapter 7, Blackwell, 2003, p.210-219.

Lukes, S Power: A Radical View, London : Macmillan. 1973, 2ème Ed 2005

Marx, K. Theses sur Feuerbach, PUF, 1987, 1èr edition 1848.

Meyer, J., et Rowan, B. "Institutionalized Organizations: Formal Structure as Myth and Ceremony”, American Journal of Sociology, Vol 83, n², 1977, p.340-363.

Peng, M.W, Business Strategies in Transition Economies. Sage Publications, 2000.

Pfeffer, J. et Salancik, G.The External Control of Organizations, Harper \& Row, 1978.

Rancière, J. La Mésentente, Politique et Philosophie, Paris, Galilée, 1995.

Rancière, J. «Politique, identification, subjectivation» in Aux bords du politique. Paris, Gallimard Folio Essais, 1998.

Scott R., Institutions and Organizations, Thousand Oaks, CA: Sage, 2n Ed, 2001.

Scott, R. Organizations: rational, natural and open systems, Pearson Education, Upper Saddle River, New Jersey, Prentice Hall, 5ème edition, 2003.

Seo, M-G., and Creed, W.E.D. "Institutional Contradictions, Praxis and Institutional Change : A Dialectical Perspective”. Academy of Management Review Vol. 27, n², 2002, p. 222-247

Simmel G, The sociology of Georg Simmel, New York, Free Press, 1950.

Swidler, A. Talk of Love. How Culture Matters. University of Chicago Press, 2001.

Tolbert, P. S. \& Zucker, L.G. "Institutional sources of change in the formal structure of organizations: The diffusion of civil service reform, 1880 - 1935 ». Administrative Science Quarterly, 1983, Vol 28, p.22-39.

Weber M., Economie et Société, T.1. Plon, 1995, $1^{\text {ère }}$ edition 1922,

Westphal, J.D., Gulati R. et Shortell S.M. "Customization or Conformity ? An Institutional and Network perspective on the Content and Consequences of TQM Adoption", Administrative Science Quarterly, 42, 1997, p.366-394. 
Zucker, L.G. « The role of institutionalization in cultural persistence. » American Sociological Review, Vol 42, 1977, p. $726-743$.

Zuckerman, E. "The categorical imperative: Securities analysts and the illegitimacy discount." American Journal of Sociology. Vol 104, 1999, p.1398-1438. 


\section{Annexe 1 \\ Les trois dimensions du pouvoir chez Steven Lukes}

En partant des travaux antérieurs, notamment de Dahl, et de Bachrach et Baratz, Lukes $(1974,2005)$ propose une présentation tripartite du pouvoir

La première dimension renvoie à l'influence directe et ouverte qu'un acteur a sur un autre. A a du pouvoir sur B quand il peut faire faire à B quelque chose que B n'aurait pas fait autrement, B étant conscient que cela vient de l'action de A. Les conflits liés au pouvoir sont ouverts.

La deuxième dimension concerne la non-décision. A a du pouvoir quand il parvient à ce que les valeurs sociales et politiques, les pratiques institutionnelles limitent l'étendue des problèmes publics traités à des problèmes qui ne posent pas de problèmes à $\mathrm{A}$. Le pouvoir s'exerce dans la définition de l'agenda politique (ce qui doit, plus exactement ce qui va, être traité ou ne doit pas l'être)

La troisième dimension du pouvoir renvoie au pouvoir invisible consistant à déterminer les préférences des acteurs. Il s'agit d'empêcher les individus, à quelque degré que ce soit, d'avoir des griefs en façonnant leurs perceptions et leurs préférences de telle sorte qu'ils acceptent leur rôle dans l'ordre social, soit parce qu'ils ne peuvent imaginer d'alternative, soit parce qu'ils le considèrent naturel et immuable. Cette troisième dimension entend rendre compte des formes les plus insidieuses de pouvoir, notamment celles fondées sur la domination lorsque les dominés acceptent leur domination, soit par résignation, soit par adhésion aux valeurs qui la fondent. 